\title{
Trilogía de Dolan: ¿dos o tres?
}

\author{
Xavier Dolan | Maté a mi madre | 2009 | Amores imaginarios | 2010| Laurence de todos modos | 2012
}

\author{
Nicolas Rabain*
}

Aix Marseille Université

Recepción: 15 de diciembre de 2019; aceptación: 2 de febrero de 2020

\begin{abstract}
Resumen
A partir de las tres primeras películas dirigidas por Xavier Dolan - Yo maté a mi madre (2009), Los amores imaginarios (2010) y Laurence anyways (2012) -, abordaremos el resurgimiento de los fantasmas matricidas en la pubertad. Si el asesinato del padre constituye uno de los pilares del psicoanálisis, el matricidio remite tanto a fantasmas edípicos como arcaicos. ¿Cómo lograr desprender las investiduras del objeto primario y destinarlas a objetos de substitución en la adolescencia? ¿Sobre quién sostenerse para alejar a una madre de mil caras: dedicada, paraexcitante, primer espejo del ser, amorosa y amada; pero también seductora, intrusiva, abusiva y traicionera, que puede, alternativamente, invadir y abandonar... En Dolan, el odio de la madre es crudo, masivo e incontrolable. Proviene del exceso de lo sexual infantil que viene a obstaculizar el trabajo de la represión. De hecho, no permite ninguna separación y hace de la progenitora una divinidad sobrehumana, todo-poderosa, aterradora, al resistir a la agresividad de su hijo con tanta tenacidad y éxito.

Si la trilogía de Dolan bordea toda lógica binaria hetero-normativa -al poner en escena recorridos subjetivos singulares lejos de todo estereotipo-, subsiste de todas formas un punto ciego: la transformación progresiva de los lazos a la imago materna. Odiar a la madre va de la mano con la imposibilidad de abandonarla. El precio: una lucha desenfrenada contra la angustia, la amenaza depresiva e incluso el derrumbe melancólico. Es el hijo, su madre y nadie más. Con esta coyuntura, ¿cómo podría tener lugar la guerra de Tres?
\end{abstract}

Palabras clave: Complejo de Edipo | Intergeneracional | Matricidio | Transidentidad

Dolan's trilogy: two or three?

\begin{abstract}
Based on the first three movies directed by Xavier Dolan - I Killed My Mother (2009), Heartbeats (2010), and Laurence anyways (2012) we will look at the resurgence of matricidal fantasies during puberty. If the killing of the father is one of the cornerstones of psychoanalysis, that of the mother refers to oedipal and archaic fantasies. How can we succeed in disinvesting the primary object in favour of substitutes during adolescence? On whom can we rely to keep at arm's length a mother with a thousand faces: devoted, protective, first mirror of the self, loving and beloved; but also seductive, intrusive, abusive and treacherous, who, in turn, invades and abandons? In Dolan's work, the hatred of the mother is raw, massive and irrepressible. It stems from the child's excessive sexuality, which thwarts the primal repression. Indeed, separation is not allowed in such context that makes the mother a superhuman, omnipotent, frightening deity who resists with tenacity and success to the son's aggressivity.

If Dolan's trilogy bypasses the hetero-normative binary logic - by staging singular subjective journeys far from any stereotype -, a blind spot nevertheless remains: the progressive reshaping of the links to the maternal imago. Hating one's mother goes hand in hand with the impossibility of leaving her. The price to pay: a frantic struggle against anxiety, the threat of depression, or even collapse. It's the son, his mother and nobody else. In such a configuration, how could any triangulation take place?
\end{abstract}

Keywords: Intergenerational | Transidentity | Matricide | Edipus complex

Tomando como punto de partida Matricidio, nombre que Xavier Dolan quería dar al primer largometraje que realizó cuando tenía dieciséis años, pensábamos escribir fundamentalmente sobre la madre. No será así. Frente a su obra, como ocurre con la obra de Winnicott, los comentarios más frecuentes -y los más superficiales- convergen muy a menudo en una misma conclusión, de las más simplistas: “'Se olvidó del padre!”. No tomaremos esos caminos. Este trabajo tratará tanto sobre la madre como sobre el padre, omnipresente en el vacío. Intentaremos mostrar hasta qué punto la obra cinematográfica del joven director quebequense puede aprehenderse también como un llamado vehemente dirigido a un padre que se aleja, a un padre que puede llegar a desaparecer completa y definitivamente, aunque esté todavía al alcance de la mano. ¿Cómo puede un hijo llamar la

*nrabain@hotmail.com 
atención de su progenitor? ¿Cómo puede hacerse notar? ¿Cómo puede diferenciarse de la madre, de quien no se ha separado lo suficiente pero, aún peor, al campo de quien fue relegado demasiado rápidamente? Intentaremos mostrar la complejidad de la relación con un padre que se idealiza y evita a la vez, por el gran temor que se le tiene.

Sin embargo, antes de considerar las relaciones con la imago paterna, abordemos primero las representaciones maternas tal y como aparecen en el comienzo de la obra de Xavier Dolan. Claramente, todo en ella lo exaspera: le desagrada su manera ruidosa de comer, así como le repugna la comida que le queda en la comisura de los labios. Y no está equivocado. El propio espectador se ve atravesado por esa misma aversión gracias a un encuadre ajustado y centrado en una boca manchada con restos de queso. De este modo, nos vemos transportados a nuestra propia adolescencia y a los horribles silencios rotos por la deglución obscena de nuestros padres de entonces, alrededor de la mesa del comedor. Pero ¿por qué diablos semejante ruido? ¿Tendremos algo que ver con esa horda de primitivos que hablan con la boca llena y que muestran con tanta indecencia el contenido de su cavidad bucal, llena y atestada de dientes que saltan a la vista mientras trituran ruidosamente alimentos irreconocibles, sucios, antes de que el bolo alimenticio se adentre en el esófago en dirección al recto? ¡Inmunda abyección!

Esa escena inaugural del primer largometraje de Xavier Dolan, Yo maté a mi madre (2009), instala el decorado de modo categórico. Muy lejos de una captatio benevolentice, vemos en él una propuesta identificatoria diacrónica, ya que el joven director interpela de entrada a los adolescentes que supimos ser. Dentro de ese período -memorable para cada uno de nosotros, pero relativamente poco accesible debido a la represión secundaria-, Dolan agrega una situación específica: el insoportable espacio cerrado sin salida en el que se encuentra un hijo solo con su madre. Ambos viven en un departamento oscuro, confinado, cubierto de telas dudosas y rodeado de pieles de animales. En resumen, el reino del kitsch y del mal gusto. Detrás de las pantallas de tela de leopardo, ¿es posible que en la madre de Hubert duerma una tigresa? Al estar divorciada y, a priori, no tener amante, está enteramente disponible para su hijo único. Una "vieja arpía”, en cierto modo, que todos los días lo lleva al liceo y deja que le grite: “¡Te odio!”. Él le repite sin cesar: “¡Me das asco! ¡Estás totalmente 'Alzheimer’!”. Por un lado, un imbécil, por otro, una mujer desmesuradamente resignada, ese es el cliché instantáneo que esboza el punto de partida de la obra de Xavier Dolan y que será nuestro tema del día.

A partir de sus tres primeras películas -Yo maté a mi madre (2009), Los amores imaginarios (2010) y Laurence Anyways (2012)-, intentamos detectar los diferentes obstáculos que encuentran los protagonistas a la hora de reestructurar sus relaciones con sus imagos parentales: aparecen la adolescencia y el ineludible resurgimiento de los fantasmas edípicos -por lo tanto, parricidas e incestuosos-. Si el asesinato del padre constituye una de las piedras angulares del psicoanálisis, el matricidio, por su parte, tiene la particularidad de remitir tanto a fantasmas edípicos como al registro arcaico. Tomando en cuenta esos dos ejes, ¿en qué consiste, entonces, la desinvestidura [désinvestissement] del objeto primario a favor de objetos de substitución en la adolescencia? Asimismo, cuando el padre brilla por su ausencia en la realidad externa, y resulta poco accesible en la realidad interna, ¿en qué instancia apoyarse para poner distancia ante una madre de mil caras: primer espejo del ser, dedicada, amorosa y amada, pero también seductora, intrusiva, abusiva, hiperexcitante y a veces traidora, que puede, alternativamente, invadir y abandonar?

Si la relación con el padre es inconsistente, el lazo con la madre es insostenible, cualesquiera sean sus diferentes representaciones.

\section{La madre, la hija y la esposa: un tríptico que no se sostiene}

A través del protagonista de Yo maté a mi madre, Dolan intenta terminar con el objeto materno, pero la tenacidad y la constancia con las que busca dañarlo solo dan cuenta de la intensidad del amor que tiene por él y que intenta a toda costa contrainvestir [contre-investir].

En Los amores imaginarios, se opera una tentativa de triangulación: Marie y Francis, amigos fusionales, se enamoran ambos del mismo efebo, Nicolas, un joven Narciso que remite al Querubín de las Nozze di Figaro, o incluso al visitante interpretado por Terence Stamp en Teorema (1968) de Pasolini; en suma, un ser ante cuyos encantos cualquiera sucumbiría. En esta segunda película, a los dos amigos, inseparables, cómplices y solidarios, les fascina el mismo personaje de sonrisa tan angelical como enigmática. De este modo, comparten un objeto común, a falta de desearse el uno al otro. Resultado: ni Marie ni Francis captan la atención de Nicolas, puesto que los dos acólitos parecen constituir las dos 
caras de una misma moneda. Nunca Marie sin Francis; nunca Francis sin Marie. Así, Nicolas se dirige sistemáticamente a ambos a la vez antes de rechazarlos en el mismo momento, tras una cachetada que Marie le da a Francis. Ese agravio marca el fin del trío, si es que hubo trío; ya que, en el fondo, nada logrará escindir la formidable díada. Esto nos hace pensar en el discurso sobre el amor de Aristófanes en El Banquete de Platón, y más precisamente en el mito del andrógino que pone en escena entidades esféricas, impertinentes de perfección. La mayoría de las veces, están constituidas de una parte femenina armoniosamente complementaria a una parte masculina. Solo la intervención de Zeus habría logrado desunirlas y generar el deseo ardiente e imperioso que cada mitad experimenta hacia su otra mitad perdida. Ahora bien, en Dolan, la separación no existe, al menos no la suficiente.

De este modo, en esas dos primeras películas, un chico y una mujer soltera son abandonados al mismo tiempo por un hombre que ya no está allí: el padre en $Y o$ maté a mi madre; el chico presumido de Los amores imaginarios, que terminará escapándose ocho meses a Asia. Cuando vuelve del viaje, este se topará con Francis y Marie, que lo rechazarán con firmeza, solidarios e impasibles. ¡Exit, Adonis rubio! Don Juan está muerto, ¡que viva Don Juan! Lo que da lugar a un nuevo seductor, morocho esta vez, interpretado por Louis Garrel. Incluso antes de que haya podido iniciarse un proceso cualquiera de desidealización, el ciclo puede volver a comenzar: "Recordar [tal vez], repetir [seguramente,] [pero sin] reelaborar" (Freud, 1914).

Dos años más tarde aparece Laurence Anyways, que plantea el siguiente enigma: un hombre que sigue amando y deseando a su mujer cambia de género progresivamente. Una vez más, el hombre heterosexual se volatiliza para transformarse aquí en mujer transgénero homosexual: Xavier Dolan, desde 2012, concientiza a su público sobre la identidad de género, $y$, más particularmente, sobre cuestiones transidentitarias: se puede nacer hombre y desear a las mujeres, y luego querer convertirse en una mujer que desea, también ella, a las mujeres. Dicho de otro modo, los hombres que usan medias de nylon no son necesariamente homosexuales, a diferencia de lo que la gran mayoría presume erróneamente.

Así, entre los numerosos hilos conductores de la trilogía de Dolan, señalamos el "declive” o la "desaparición” aparente -es decir, el "Untergang"- no del complejo de Edipo sino del hombre heterosexual. "Der Untergang” implica, primero, volar en pedazos, luego resurgir con otra forma: un padre que abandona a su esposa y su hijo en Yo maté a mi madre; un seductor que deja de interesarse en un chico y una chica tras haberlos cautivado, o más bien hechizado, en Los amores imaginarios; luego, un marido amante que se convierte progresivamente en una esposa extenuada en Laurence Anyways. Entonces, en la obra de Dolan, la pregunta no es "¿qué quieren las mujeres?”, sino "¿qué quiere el padre?”. ¿Por qué huye cobardemente? ¿Por qué capitula tan rápido? ¿Cómo volver a encontrarlo y, sobre todo, cómo retener su atención? ¿Cómo construir con él un lazo satisfactorio? ¿Cómo asegurarse de su fiabilidad? ¿Cómo saber si no terminará cansándose de su vida familiar y navegando hacia nuevos horizontes? Para sobrevivir a esa discontinuidad y encontrar en la imago paterna un apoyo en vez de un lugar vacante, volvamos a lo que Winnicott (1971) evoca al final de su obra: el "choque de las armas" (p. 200) ineludible.

\section{La confrontación entre las generaciones}

Algunos críticos de cine consideran a Dolan como un imbécil o un niño mimado. Para nosotros, es más bien un carenciado de lo paterno, ya que fue abandonado por los hombres de la generación que precede a la suya. El cineasta erra por los caminos áridos de la búsqueda de un padre no solo amoroso y narcisísticamente valorizador, sino también de un rival edípico al cual enfrentarse, lo que nos lleva a pensar en La confrontación generacional (2018), el único libro de Luis Kancyper traducido al francés. El título de la obra promete destacar la noción de confrontación intergeneracional que el autor erige como operador y condición indispensable para el proceso de subjetivación. En ese libro, Kancyper diferencia tres situaciones:

- Primera configuración, y es el único caso favorable: la confrontación intergeneracional da lugar a un desapego progresivo entre los adolescentes y sus padres. La dinámica edípica orquesta mayoritariamente los conflictos psíquicos subyacentes. Se trata de una confrontación llamada trófica en la medida en que favorece el proceso de subjetivación. En Dolan, no encontramos nada de esto.

- Segundo caso posible: la confrontación entre las dos generaciones no contribuye a una mayor autonomía de una en relación con la otra. En este caso, la preponderancia de los lazos de naturaleza narcisista da origen a una confrontación de naturaleza mortifera. Esto nos remite a los conflic- 
tos entre Hubert y su madre en la primera parte de la trilogía: los alaridos incesantes, sin ningún reordenamiento de su relación.

- Tercera y última situación: la ausencia de confrontación. En este último caso, los adolescentes y sus padres huyen de los conflictos, eteramente evitados. En esta situación podemos ubicar a Dolan y su padre.

Kancyper agrega que algunos padres no facilitan la aparición de sentimientos hostiles, por diferentes razones que no desarrollaremos aquí. Al impedir el nacimiento de una oposición en sus hijos, esos adultos perjudican al mismo tiempo la dialéctica intergeneracional necesaria para el proceso de separación. Esa negación del odio y de la ambivalencia remite a los dos tipos de educación patógena que identificó Freud (1930): la severidad excesiva y el laxismo. Aunque sean opuestos, en ambos casos el yo no se diferencia lo suficiente de los objetos parentales: "El padre 'desmedidamente blando e indulgente' ocasionará en el niño la formación de un superyó hipersevero, porque ese niño, bajo la impresión del amor que recibe, no tiene otra salida para su agresión que volverla hacia adentro" (p. 318). Ese superyó feroz es recurrente en Dolan y sus protagonistas, que son sus fervientes herederos: siempre listos para autocastigarse, para trabajar con ahínco temiendo no estar nunca a la altura, sean cuales sean los éxitos que puede reflejar la realidad externa. Xavier Dolan, con treinta años, tendrá pronto ocho películas premiadas; cientos de miles de fans de todos los bandos, de todos los horizontes, que no bastan en absoluto para calmar su sed de reconocimiento.

Junto a esa tendencia a volcar su propia agresividad hacia sí mismo, observamos una diferenciación insuficiente entre el yo y el objeto, y una problemática edípica con dificultad para elaborarse, si no es en estado embrionario. Según la perspectiva kleiniana, podríamos sostener que la mayor parte del tiempo estamos por fuera de la posición depresiva. La mayor parte del tiempo, el objeto está dividido: en Yo maté a mi madre, el pecho bueno se materializa en una docente de literatura que alimenta a Hubert con citas de textos románticos y poesía. Ella también fue abandonada por su padre diez años antes y vive sola (la actriz es Suzanne Clément). El objeto malo, por su parte, está encarnado por la madre real que $\mathrm{Hu}-$ bert decreta casi tan loca como su abuela materna: "Hace diez años que no estás con un tipo porque sos igual a tu madre, ¡no sos más que una loca!”. En Laurence An- yways, la abuela materna también sigue un tratamiento psiquiátrico. En Dolan, la locura parece transmitirse de madres a hijas.

Sin embargo, el objeto materno no siempre está dividido ni genera siempre angustias de persecución. Una noche en la que se drogó, Hubert entra en la habitación de su madre. La despierta para soltarle un discurso frenético e incoherente del que solo podemos retener una información: jama a su madre más que a nada! Una escena de a dos, como siempre o casi; la presencia de un tercero es rara, con excepción de las grandes fiestas en las que se excita al espectador con la música que no deja de generar una exaltación sensorial fuera de lo común. El erotismo de los cuerpos que festejan está en el centro de la pantalla, reforzado por un trabajo cromático. Un calibrado que vuelve a los colores primarios sirve como vector de sensaciones: el rojo, el azul, el amarillo, pero también el verde, están al servicio de los amores plurales en los que los cuerpos se besan con gran pudor. No hay escenas sexuales tórridas en Dolan. La intensidad está en todos lados y suele ser masiva, pero siempre se mantiene fuera de las escenas de amor sensual en las que, una vez en la cama, los personajes parecen más bien atrapados por el hastío, cuando no presos de angustias existenciales.

Así, las escenas con más de dos personajes son casi inexistentes, o se acaban con bastante rapidez. En definitiva, "siempre hay dos sin tres".

\section{"Siempre hay dos sin tres"}

En Yo maté a mi madre, a Hubert se lo filma sentado a la mesa con su madre de forma inhabitual y audaz: el marco insiste en el vacío. Mientras que los dos están alrededor de una mesa y ocupan todo el espacio, un lugar vacante se ubica en el medio del salón. El encuadre le da una importancia mayor en tanto apertura hacia el exterior, just in case...

Si tres personas cohabitan en un mismo espacio, nunca se las filma juntas. Sucede lo mismo con la circulación de la palabra. Cuando a Hubert lo atrapan sus padres, separados pero excepcionalmente reunidos para enviarlo a un internado, el malestar es paroxístico. Se enfurece: “¿De dónde saliste? ¿Trabajás, reaparecés y ahora te hacés cargo de tu deber de padre y me arruinás la vida? ¡Fuck you!”. Todo sucede como si lo que hubiera podido tomar la función de tercera instancia solo interviniera sistemáticamente con intermitencia y como si toda 
perspectiva de cambio perenne se viniera abajo inevitablemente.

Otro malestar que generan las situaciones de a tres: en la casa del novio de Hubert, la madre, bastante inmadura, mantiene con su hijo un lazo excepcional, de naturaleza casi fraternal. Ninguna confrontación intergeneracional es posible en una configuración como esa. Hubert se mantiene al margen, incómodo frente a una imago materna de nuevo peligrosa por ser complaciente y particularmente seductora con su propio hijo.

En Laurence Anyways, la madre, interpretada por Nathalie Baye, primero está dedicada por completo a su marido postrado y parapléjico; ese cuadro siniestro se completa con un síndrome frontal resultado de un ACV. Al principio, la madre rechaza la transidentidad de su hijo hasta que la situación da un vuelco. Totalmente fuera de sí, un día destroza la pantalla del televisor de su marido, como un Moisés que rompe las Tablas de la Ley. Se trata de un nuevo inicio en el que abandonará a su marido en beneficio de su hijo: "No sentía que fueras mi hijo, pero ahora siento que sos mi hija”. Del marido ya no se hablará más.

En cuanto a Laurence, lo vemos ya sea en compañía de su madre, ya sea con su esposa, Fred. Señalemos al paso el uso de nombres epicenos en la película, debido a la disforia de género: Laurence y Fred. Asimismo, Fred nunca se cruzará con la madre de Laurence. Todo sucede en dúo, en duelo, en díada. Cada amor se convierte en totalitario y sofocante de un modo inevitable. Es cuestión de tiempo. ¿Significa eso que no hay ninguna reestructuración identificatoria en curso y que las imagos parentales se mantienen fijas?

\section{Reestructuraciones imagoicas e identificatorias}

Las psicoterapias analíticas de adolescentes y la mayoría de las películas de Xavier Dolan constituyen dos lugares específicos de un mismo proyecto: la desinvestidura de los objetos parentales a favor de objetos de substitución, con miras a una salida exogámica. ¿Cómo se reestructuran los lazos con los objetos parentales en la pubertad, es decir, en un momento en el que lo infantil vuelve a aparecer? ¿En qué medida la reinvestidura [réinvestissement] de ese infantil conduce a reactivar el proceso de subjetivación en algunos casos y a dificultarlo en otros?

Traigamos a colación "la novela familiar de los neuróticos” (Freud, 1909). Del mismo modo que, en sus fan- tasmas, el niño reemplaza a sus propios padres por progenitores "de una posición social más elevada” (p. 218), el adolescente suele estar dispuesto a desinvestir a sus padres reales a favor de figuras identificatorias percibidas como más nobles, como la profesora de francés en Yo maté a mi madre, quien se fascina a su vez por la madurez de su alumno. Más allá de la complicidad intelectual, comparten un drama común: la huida del padre frente a sus responsabilidades, pero también su aparente indiferencia y su capacidad para sobrevivir, a priori sin ningún problema, al distanciamiento con respecto a sus hijos. Con él, las interacciones se agotaron; Hubert y su profesora se encariñan, como dos compañeros de desgracias. ¿Acaso esta última no encarna, por varios motivos, la buena madre, dulce, cariñosa y que debe ser consolada? En ese sentido, ella también forma parte de Hubert... En definitiva, siempre volvemos al mismo punto: en Dolan, el otro nunca es por completo un objeto externo, sino más bien una parte de sí, buena o mala según el caso, que se puede amar u odiar.

¿Odiar antes de desinvestir? Desde el punto de vista económico, la desinvestidura es uno de los principales mecanismos ligados a la operación de represión que implica "un juego complejo de desinvestiduras, reinvestiduras y contrainvestiduras" (Laplanche, Pontalis, 1967, p. 396). Si el movimiento exogámico implica cierto número de operaciones como la desinvestidura parcial de los objetos parentales, y también su desidealización, el odio mantiene desde luego un lugar central en el proceso del proyecto. Ahora bien, en Dolan el odio parece "circunscribir su objeto y aferrarse a él más que a nada" (André,Bernateau, 2004). En ese caso, ¿el odio no desvía de la desinvestidura? En otros términos, ¿cómo puede el odio constituir un incentivo para la separación si provoca una reinvestidura del objeto con tanto fervor?

Freud (1988) recuerda que "el odio es, como relación con el objeto, más antiguo que el amor” (p. 186). Desde una perspectiva preedípica, el odio se ve entonces "reforzado por la regresión del amar a la etapa sádica previa, de suerte que el odiar cobra un carácter erótico y se garantiza la continuidad de un vínculo de amor" (p. 186). Freud asocia el odio al refuerzo de la frontera entre el yo y el mundo exterior: "Lo exterior, el objeto, lo odiado, habrían sido idénticos al principio. Y si más tarde el objeto se revela como fuente de placer, entonces es amado, pero también incorporado al yo, de suerte que para el yo-placer purificado el objeto coincide nuevamente con lo ajeno y lo odiado” (p. 183). En este punto, Winnicott (1975) afirma que "la destruc- 
ción desempeña su papel al fabricar la realidad, al ubicar al objeto por fuera de sí” (p. 126). Si odiar a la madre da cuenta de la necesidad de diferenciarse, de separarse de ella, nada garantiza, en cambio, que la empresa tendrá éxito.

En la adolescencia, como sostiene Winnicott (1971), la ejecución simbólica de la madre está sustentada por un fantasma de asesinato allí donde, durante la infancia, se trataba de un fantasma de muerte. Esto nos lleva a volver a analizar la noción de matricidio a la luz de las películas de Dolan.

\section{El matricidio en Dolan}

El matricidio es una empresa doble, necesariamente: implica el asesinato de la madre arcaica y el de la madre edípica. En este sentido, la obra de Dolan se abre con los labios de la madre. Permanecer indiferente ante la boca de su progenitora le es totalmente imposible. Todo ocurre como si la imagen del pecho malo se hubiera hallado en fantasmas evocadores de voracidad o de miedo a la voracidad. En este sentido, el primer plano centrado en la boca de la madre remite a la imagen que el bebé tiene de su madre cuando esta lo tiene en brazos. Winnicott sostenía que el bebé se ve en la mirada de la madre, pero ¿qué ve cuando contempla su boca, que por si fuera poco está masticando? Melanie Klein (1966) afirmó que "el pecho malo va a devorar al niño con una voracidad igual a la de los deseos de este" (p. 197). La relación del bebé con el pecho bueno se opone a esas angustias de devoración. A esas angustias de devoración y de engullimiento se suman las angustias de persecución propias de la posición esquizoparanoide. Por su parte, la posición depresiva se mantiene en estado de esbozo, como demuestran las tentativas de reparación transitoria de Hubert: ordenar de punta a punta el departamento cuando su madre está ausente, como un ama de casa perfecta. No bien regresa la madre, las peleas no tardan en reaparecer con más fuerza.

En el plano edípico, Green (1992) sostiene que, en la pubertad, "la sexualidad infantil, cuya accesión al Edipo fue el término provisorio, apunta a una nueva realización del Edipo una vez que este se disolvió” (p. 232). El resurgimiento de los anhelos edípicos reviste un valor paradigmático en la adolescencia. La madre que debe eliminarse también es la madre edípica en la perspectiva del Edipo llamado "negativo" o "invertido", es decir, el obstáculo para obtener el amor del padre. Pero en
Dolan ninguna triangulación se sostiene. Respecto de este punto, a algunos les ha parecido adecuado evocar el concepto greeniano de "bi-triangulación", que opone una imago materna aterradora con una imago paterna preservada e idealizada, es decir, exenta de toda crítica, lo que no va para nada en nuestro sentido, ya que para nosotros el padre solo está preservado de críticas en apariencia. En este tipo de relación de objeto, Green (1990) sostiene que:

creemos que nos enfrentamos a una relación triangular que conlleva una distinción entre las dos figuras parentales, la madre y el padre, pero en lugar de sentimientos ambivalentes, positivos y negativos a la vez con respecto a cada uno de los padres, hay una división entre el bueno y el malo, el perseguidor y el idealizado, un padre totalmente maléfico y otro totalmente benéfico. El buen padre es ineficaz frente al malo, su debilidad e idealización excesivas no son de ninguna ayuda para enfrentar la 'maldad' omnipotente del otro. Todo ocurre como si la relación de objeto, conectada con las pulsiones, estuviera tan cruelmente colmada de destructividad que la única manera de combatirla fuera edificar una relación de objeto narcisista idealizada que se revela necesariamente deficiente. (p.159)

\section{Conclusiones}

En cualquiera de las partes de esta trilogía de Dolan, estamos muy lejos del número tres. Y, como señaló Winnicott (1958), “1+1 es 1". En Yo maté a mi madre, son la madre y el hijo; el padre está fuera del juego y solo aparecerá una vez en medio de la violencia. En Los amores imaginarios, se trata de la relación gemelar entre un chico y una chica que comparten un fantasma desmesurado con respecto a un desconocido. En Laurence Anyways, es el "Macho a Hembra" y su esposa que lo acompaña en su recorrido de transición. En cada una de las tres películas, el hombre heterosexual no manifiesta ningún interés particular por los protagonistas. Sucede lo mismo con las películas posteriores. En Mommy, se trata nuevamente de una madre y un hijo que no deja que nadie se acerque a ella. En The death and life of John F. Donovan, encontramos nuevamente una pareja madre/ hijo. La madre nunca mira a los hombres; dicho de otro modo, permanece como una Virgen María a la que solo su hijo puede acceder.

Aunque el odio a la madre es crudo, masivo e irreprimible, originado en la desmesura de lo sexual infantil que 
vino a frustrar el trabajo de la represión, no permite de hecho ninguna separación. Hace de la progenitora una divinidad sobrehumana, todopoderosa, temible por resistir con tanta tenacidad la agresividad de su hijo: "No puedo vivir más con vos... ¡me arruinás la vida! Solo quiero escaparme; escaparme a un desierto; cavar un pozo en la duna, sin aire, sin agua, sin nada; ipero al menos, me habré librado de vos!", asegura Hubert. Pero entonces, ¿cómo puede ser que el adolescente no se vaya a vivir a la casa de su novio, que se lo propuso en varias ocasiones? Hubert prefiere quedarse bajo el techo de un objeto parental odiado del que, sin embargo, no logra alejarse: ingrato ante quien lo crio y, paradójicamente, incapaz de liberarse; dividido entre la sed de libertad y una necesidad incomprensible de regresar a quien dice detestar. Odiar a la madre va de la mano con la imposibilidad de dejarla. El precio: una lucha desenfrenada contra la angustia, la amenaza depresiva, incluso el derrumbe melancólico. Es el hijo, su madre y nadie más. Todo ocurre como si no pudiera haber guerra "de tres". ${ }^{1}$

En el final de este breve recorrido de la trilogía de Dolan, podemos afirmar que nos quedamos en el número "dos": el otro es más un alter ego que un objeto claramente diferenciado. Sus fronteras se confunden con las del yo. En cuanto a aquel que debería oficiar de tercero, está sistemáticamente ausente, dimisionario, escurridizo, desinvestido de sus funciones familiares. Su deseo se vuelca fuera, lejos de los protagonistas, lejos de la díada. En definitiva, el tercero no apareció y el director intenta contra viento y marea ser reconocido, investido, amado por una entidad que vendría a separarlo de su madre. Tenemos a nuestra disposición una filmografía constituida de una prodigiosa serie de variaciones alrededor de un mismo tema, crístico por excelencia. En El evangelio según San Mateo, se cuenta: "Cerca de la hora novena, Jesús clamó a gran voz, diciendo: Elí, Elí, ¿lama sabactani? Esto es: Dios mío, Dios mío, ¿por qué me has desamparado?”. ${ }^{2}$ Esas son las últimas palabras de Cristo, dirigidas al Padre, antes de exhalar el último suspiro. Ese es también el punto de partida de la obra de Xavier Dolan que, como tantas otras chicas y chicos de su generación, busca desesperadamente un padre que lo ame y lo invista, y que venga a rescatarlo de un lazo desmedidamente erotizado con la madre: "Padre, ¿por qué me abandonaste? ¿Qué fuiste a buscar lejos de mí y de mi madre, tu esposa? ¿No te bastábamos, no te interesábamos? ¿Encontraste algo mejor que nosotros?”. En definitiva: "Papá, ¿por qué nos abandonaste, a mamá y a mí?”.

Traducción: Salomé Landivar

\section{Referencias}

André, J.; Bernateau, I. (2014). Argument. En Les territoires de la haine. París: PUF.

Breen, N., Grant, N., Lafontaine, L. (productores) y Dolan, X. (director). (2018). Life and death of John F. Donovan [cinta cinematográfica]. Canadá: Les Films Seville.

Corbeil, S., Dolan, X., Grant, N., Karmitz, N. (productores) y Dolan, X. (director). (2016). Es solo el fin del mundo [cinta cinematográfica]. Canadá/Francia: Lucky Red Distribuzione y Cirko Film.

Corbeil, S., Dolan, X., Grant, N., Lafontaine, N. (productores) y Dolan, X. (director). (2013). Tom en la granja [cinta cinematográfica]. Canadá/Francia: MK2 (internacional) y Entertainment One.

Corbeil, S., Dolan, X., Grant, N., Lafontaine, L. (productores) y Dolan, X. (director). (2014). Mommy [cinta cinematográfica]. Canadá: Les Films Seville Metrodome.

Dolan, X., Mondello, C., Morin, D. (productores) y Dolan, X. (director). (2009). Yo maté a mi madre [cinta cinematográfica] Canada: FilmOption International.

Dolan, X., Mondello, C., Morin, D. (productores) y Dolan, X. (director). (2010). Los amores imaginarios [cinta cinematográfica]. Canada: Alliance Atlantis.

Freud, S. (2007 [1909]). Le roman familial des névrosés. En Euvres complètes de Freud / Psychanalyse, t. VIII, dir. J. Laplanche. París: PUF, pp. 251-256 [trad. esp.: (1992). “La novela familiar de los neuróticos”. En Obras completas, traducción de José Luis Etcheverry, Buenos Aires: Amorrortu, t. IX, pp. 213-220].

Freud, S. (2005 [1914]). Remémoration, répétition et perlaboration. En CEuvres complètes de Freud / Psychanalyse, t. XII, dir. J. Laplanche. París: PUF, pp. 185-196 [trad. esp.: (1992). “Recordar, repetir y reelaborar”. En Obras completas, traducción de José Luis Etcheverry. Buenos Aires: Amorrortu, t. XII, pp.145-157].

Freud, S. (1988 [1915]). Pulsions et destins de pulsions. En Métapsychologie, Euvres complètes de Frend / Psychanalyse, t. XIII, dir. J. Laplanche. París: PUF, p. 163-187 [trad. esp.: (1992). “Pulsiones y destinos de pulsión”. En Obras completas, traducción de José 
Luis Etcheverry, Buenos Aires: Amorrortu, t. XIV, p. 105-134].

Freud, S. (2002 [1930]). Le malaise dans la culture. En Euvres complètes de Frend / Psychanalyse, t. XVIII, dir. J. Laplanche. París: PUF, p. 318, nota al pie [trad. esp.: (1992). "El malestar en la cultura”. En Obras completas, traducción de José Luis Etcheverry, Buenos Aires: Amorrortu, t. XXI, p. 126].

Gilllibert, C., Karmitz, N., Lafontaine, L. (productores) y Dolan, X. (director). (2012). Laurence Anyways [cinta cinematográfica] Canadá/Francia: Alliance Films.

Green, A. (1990 [1976]). Le concept de limite. En La folie privée. París: Gallimard, p. 121-163 [trad. esp.: (2008). "El concepto de fronterizo". En De locuras privadas, traducción de José Luis Etcheverry. Buenos Aires: Amorrortu].

Green, A. (1992). L'adolescent dans l'adulte. En Journal de la psychanalyse de l'enfant, 11, pp. 213-243 [trad. esp.: (1993). "El adolescente en el adulto”. En Psicoanálisis, APdeBA, vol. XV, n. $\left.{ }^{\circ} 1\right]$.

Kancyper, L. (2018). La confrontation entre les générations. Étude psychanalytique. París: L’Harmattan, revisión y prefacio a cargo de N. Rabain [original en español: (2003). La confrontación generacional: estudio psicoanalítico. Buenos Aires: Lumen].

Klein, M. (1966 [1952]). “Quelques conclusions théoriques au sujet de la vie émotionnelle des bébés”. En Développements de la psychanalyse, traducción de W. Baranger. París: PUF, p. 187-222 [trad. esp.: (1997). "Algunas conclusiones teóricas sobre la vida emocional del bebé”. En Obras completas de Melanie Klein, traducción de Hebe Friedenthal. Buenos Aires: Paidós].

Laplanche, J.; Pontalis, J.-B. (1967). Vocabulaire de la psychanalyse. París: PUF, 518 p. [trad. esp.: (1996). Diccionario de psicoanálisis, traducción de Fernando Gimeno Cervantes. Barcelona: Paidós].

Winnicott, D. W. (1969 [1958]). “La position dépressive dans le développement affectif normal”. En De la Pédiatrie à la Psychanalyse. París: Payot [trad. esp.: (1999). "La posición depresiva en el desarrollo emocional normal”. En Escritos de pediatría y psicoanálisis, traducción de Jordi Beltrán. Barcelona: Paidós].

Winnicott, D. W. (1969) “L'utilisation de l'objet et le mode de relation à l'objet au travers des identifications” [trad. esp.: “El uso de un objeto y la relación por medio de identificaciones”].

Winnicott, D. W. (1975 [1971]). Jeu et Réalité. París: Gallimard [trad. esp.: (2013). Realidad y juego, traducción de Floreal Mazía. Barcelona: Gedisa].

Winnicott, D. W. (1975 [1971]). “Concepts actuels du développement de l'adolescent - Leurs conséquences quant à l'éducation”. En Jeu et réalité. París: Gallimard, p. 200 [trad. esp.: (2013). "Conceptos contemporáneos sobre el desarrollo adolescente, y las inferencias que de ellos se desprenden en lo que respecta a la educación superior”. En Realidad y juego, traducción de Floreal Mazía. Barcelona: Gedisa].

1 El autor hace un juego de palabras con el título de una obra de teatro de Jean Giraudoux, La guerre de Troie n'aura pas lieu [No habrá guerra de Troya]: Troie [Troya] y trois [tres] son homófonos en francés [N. de la T.].

2 Evangelio de Mateo, 27: 46. 